\title{
Digital Smile Design, Guided Surgery, Delivering Facial Driven Implant Treatment in A Failed Dentition: Case Reports
}

\author{
Kamsiah G Haider* \\ DSD Master and Instructor, Malaysia
}

*Corresponding author: Kamsiah G Haider, Prosthodontist, DSD Master and Instructor, 15G, Jalan Wan Kadir 2, Taman Tun Dr. Ismail, Kuala Lumpur, Malaysia.

Received Date: October 27, 2018

Published Date: November 19, 2018

\begin{abstract}
The Digital Smile Design (DSD) digital implant treatment planning solutions allow for predictable prosthetic results, DSD helps in visualizing the end product through a blue print. The transition of patients from failing dentition to complete-arch implant rehabilitation often means that the patient is rendered edentulous and has to wear a removable complete denture for a period of time. Many patients find this unacceptable. With the immediate loading concept, it is possible to offer our patients a third dentition on the day of extractions. Today, many people suffering failed dentition, moving towards edentulism, getting back their smile with a fixed prosthesis could be priceless. This treatment provides a fixed interim prosthesis use throughout the rehabilitation process, allowing patient comfort and prosthodontic control. The prosthesis is supported by the immediate implants that we placed which are all on the same day. The implants and prostheses were functioning successfully after surgery. Combination of digital smile planning and guided surgery helps to make a complex case which is carefully planned simpler and predictable in their implant's placement and delivery of the prosthesis within the same day. Modern implant-based dentistry has delivered something to the edentulous patient that conventional dentures have never quite managed by comparison-substantially increased quality of life. A prosthesis held solidly in place by dental plants not only provides the comfort and security a patient is likely to be missing with removable dentures, it also preserves bone. Add to the equation emotional benefits -such as improved self-image, increased selfconfidence and a renewed social life-and an implant-based restoration becomes the clear choice.
\end{abstract}

Keywords: Digital smile design; Guided surgery; Facial driven; Implant planning and the all-on-4; Digital laboratory; 3D printing; CBCT; Decoma; STL

\section{Summary}

Background: Two case reports representing a combination of four concepts utilizing the Digital smile design, guided surgery, delivering facial driven implant planning and The All-on- $4^{\mathrm{T}}$ Procedure. Their applications are interrelated. Today, preoperative planning, and quantitative and qualitative jaw bone analysis cannot be done without the use of at one of the concepts. The latest in a series of achievements in this field is a method of making a guide for The All-on- $4^{\mathrm{TM}}$ procedure based on patient's face, utilizing the Digital Smile Design concept. This way pre implantology planning reduces the chance of prosthetic and surgical complications to a minimum and allows installation of dental implants in the most optimal position for the immediate prosthetic work [1-10].
Aim

To show benefits of facial guided implantation in clinical practice in an upper full mouth case rehabilitated with dental implants. For treatment planning in this article all challenge our willingness to accept compromise. Treatment success is most important and is subject to the interpretation of the patient. Patient satisfaction trumps the clinician's view of the ideal outcome every time that the two are not the same.

There is an increase in treatment need as the number and proportion of the elderly in the world is increasing. Available data indicates that the elderly population of Malaysia is projected to 
increase from $6.4 \%$ in the year 2000 to $7.0 \%$ in the year 2005, and subsequently to $12.0 \%$ in the year 2020 [11].

As part of the Digital Smile Design (DSD) digital implant treatment planning solutions allow for predictable prosthetic results, DSD helps in visualizing the end product through a blue print. This is unlike conventional implant placement that only considered the available jaw bone without considering the face.

\section{Case 1}

Patient Miss A, 61 years old. When we begin this project with the end in mind, we have a personal direction to guide our project, without which we cannot accomplish toward our own goals. Beginning with the end in mind is part of the process of new dentistry, with the help with the DSD protocol by taking control of the case. After that the CBCT with its qualitative and quantitative analysis of bone tissue, we then decided to digitally place the implants with straight and angled multi-unit abutments, to provide soon to be edentulous patients with an immediately loaded full arch restoration with only four implants. The software appliance made virtual implants in the most optimal positions; treatment plan was forwarded by the internet connection into the DICOM format to Digital Smile Design Laboratory in Spain, along with the STL folders of scanned plaster models in order to develop a guide. A few days later, after approval sent, we received the guide.

\section{Introduction}

Historically, the implant procedure was performed only on the basis of available jaw bone without considering the face [12].
However, with facial driven implant planning and the All-on-4 treatment concept, two implants placed vertically in the anterior region and two placed up to an angle of $45 \mathrm{o}$ in the posterior region [13]. These positions were made possible by CBCT concept of radiological diagnostics, but with improved technical characteristics of the device and accompanying software support; today, it became a gold standard in most aspect of implants diagnostics [14]. This technology allows preoperative planning and quantitative and qualitative analysis of bone before and after extractions [14].

The latest in a series of achievements in this field is a method of making a guide for implant using 3D printing technique. Prepared guide reduces surgical complications based on patients' photos and videos. All things are created twice. We create them first in with our basic DSD protocol even without treatment planning and then we work to bring them into physical existence [15].

By taking control of our own design we can edit our work thus taking control and responsibility for the outcome. We design and edit our DSD using should be considered an opportunity to achieve exceptional treatment results using alternative treatment modalities [16]. The treating clinicians and laboratory technicians in all three cases are well versed in ideal dentistry and yet feel that the outcomes in situations like these are the most fulfilling and rewarding. All three patients expressed their gratitude and appreciation for treatment that far exceeded their expectations. They were thrilled with the results and is this not the reason that we engage in our profession each day (Figure 1).

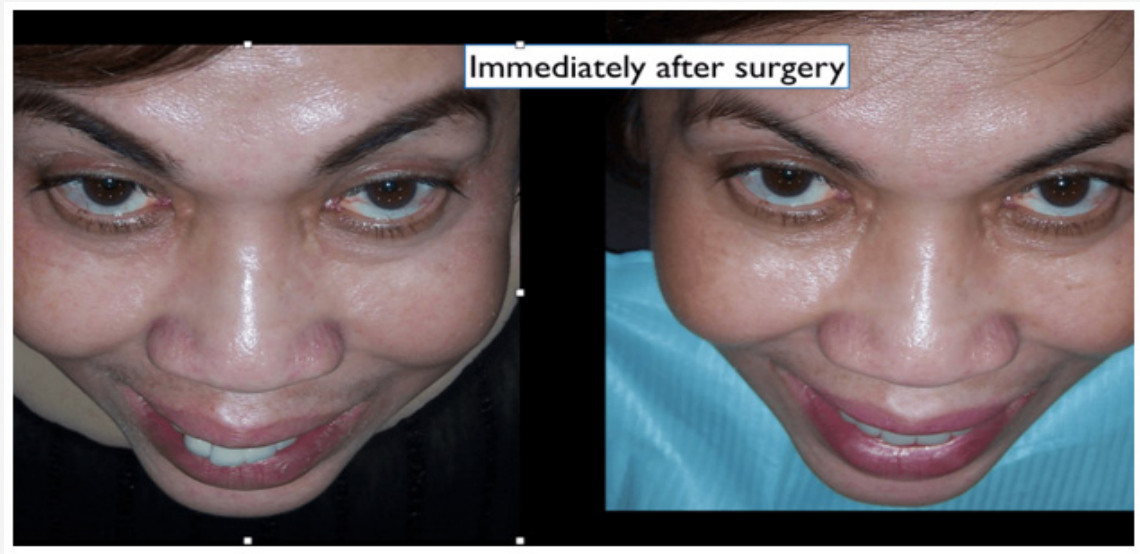

Figure 1: Photos of before and after surgery.

The transition of patients from failing dentition to completearch implant rehabilitation often means that the patient is rendered edentulous and has to wear a removable complete denture for a time. Many patients find this unacceptable [17].

Upon learning the immediate loading concept from Paulo Malo, we at Klinik Pakar Pergigian Dr. Kamsiah have been utilizing this concept. We can offer a third dentition on the same day. For many people suffering failed dentition moving towards edentulism, getting back their smile back with a fixed prosthesis could be priceless [13].
This treatment provides a fixed interim prosthesis for use throughout the rehabilitation process, allowing patient comfort and prosthodontic control. The prosthesis is supported by the immediate implants that we placed which are all on the same day. The implants and prostheses were functioning successfully after surgery [18].

\section{One True Solution}

Modern implant-based dentistry has delivered something to the edentulous patient that conventional dentures have never quite managed by comparison-substantially increased quality of life [19]. 
A prosthesis held solidly in place by dental implants not only provides the comfort and security a patient is likely to be missing with removable dentures, it also preserves bone. Add to the equation emotional benefits-such as improved self-image, increased self-confidence and a renewed social life-and an implantbased restoration becomes the clear choice $[19,20]$ (Figures 2-7).

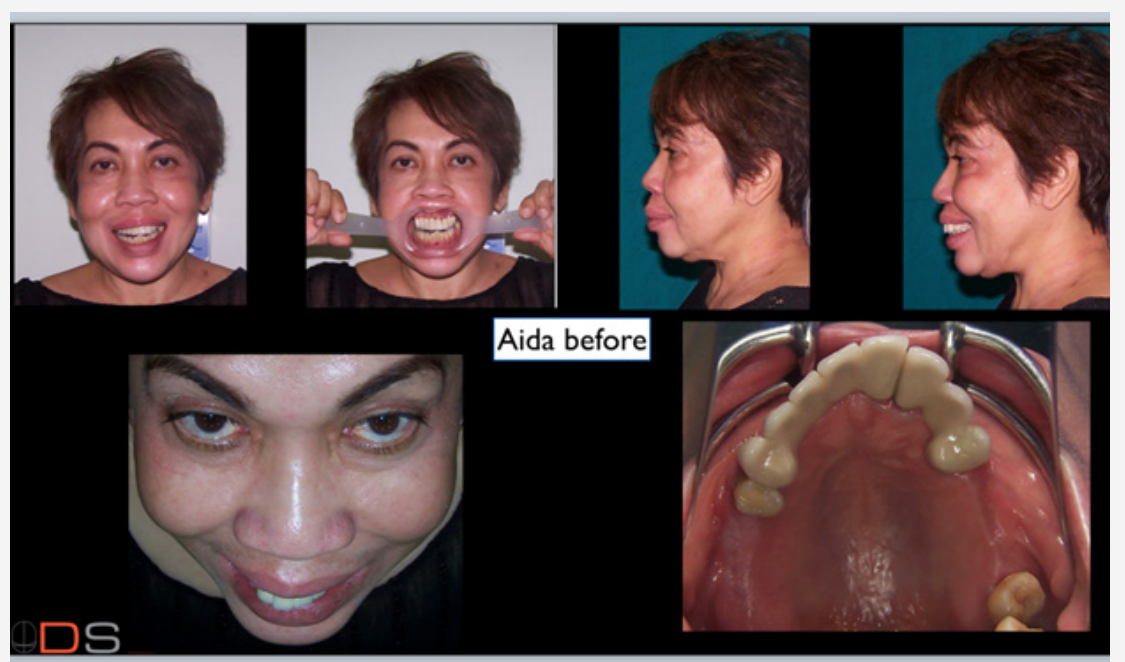

Figure 2: 6 basics photographs needed. Combining digital smile design protocol, a pre-treatment planning, diagnostics, and guided surgery with implants this situation and predictable prosthetics, as proven by numerous publications.

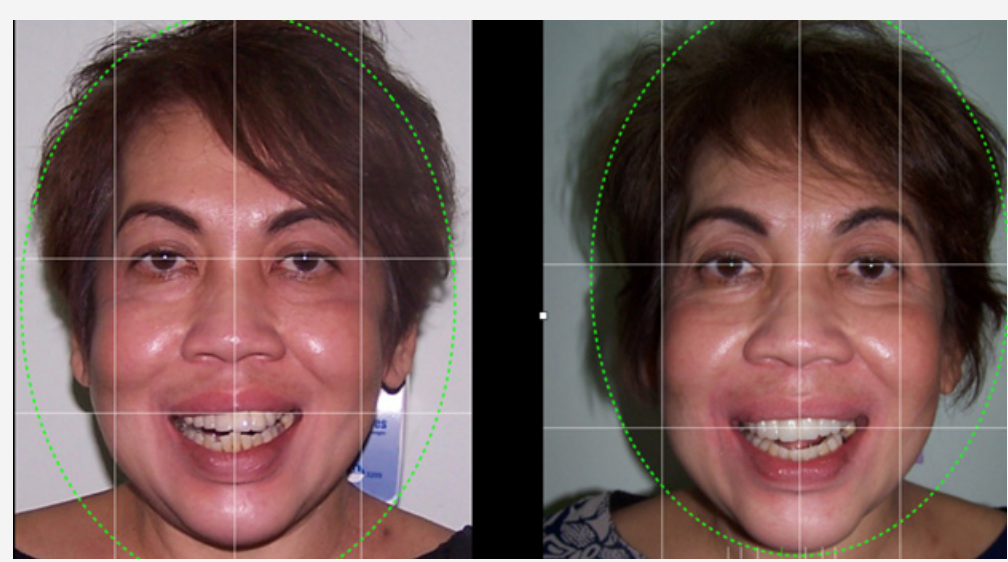

Figure 3: DSD sketches can be performed in presentation software such as Keynote (iWork, Apple, Cupertino, California, USA) or Microsoft PowerPoint (Microsoft Office, Microsoft, Redmond, Washington, USA). This improved visualization makes it easier to select the ideal restorative technique.

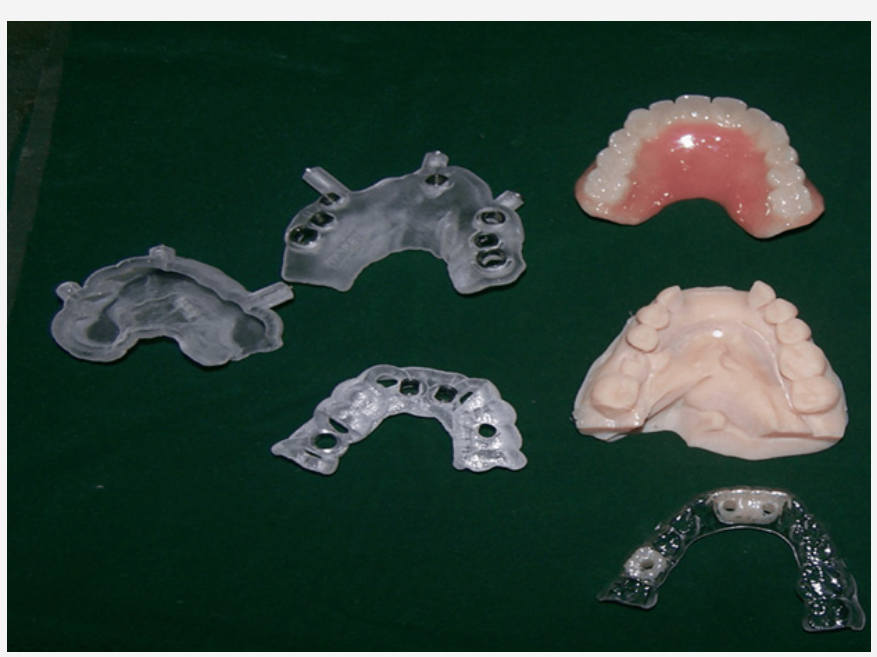

Figure 4: Guided surgery - using the DSD and a complete prosthesis as planned and facially driven treatment planning and guided implant surgery. It all come in a Magic Box. 


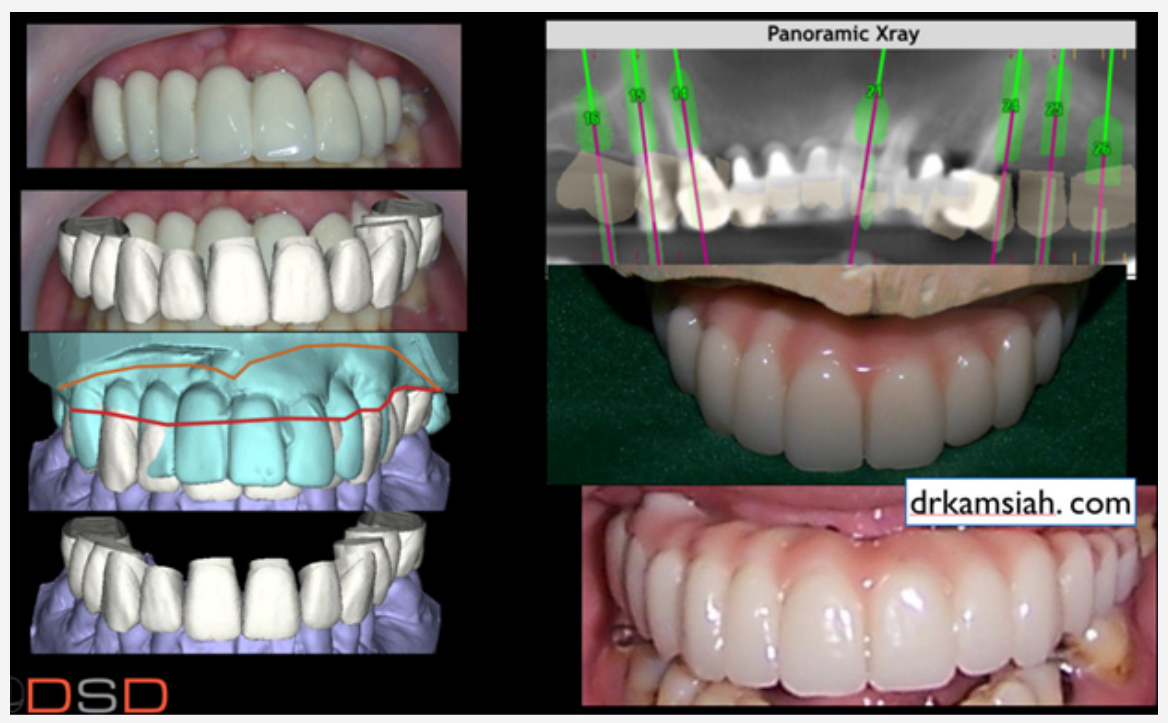

Figure 5: From the very beginning case is overall treatment was guided by the DSD protocol and its Guided surgery was carefully planned along the designed to make a complex case simple and predictable in their implants placement.

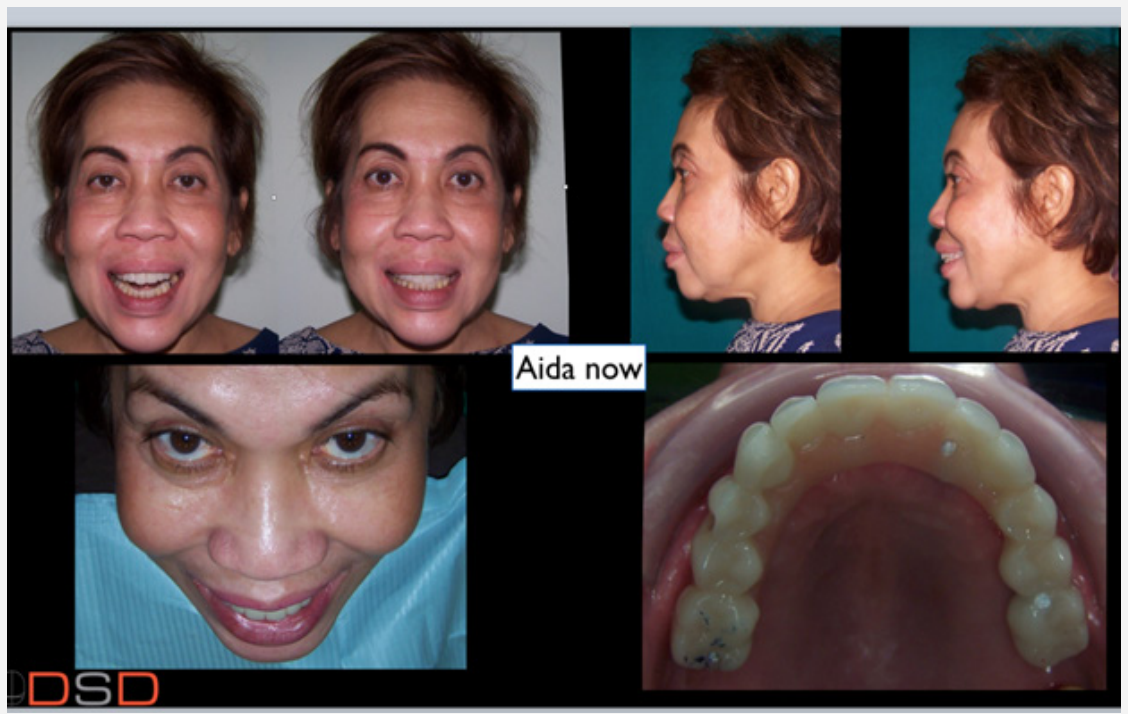

Figure 6: We need to plan the situation with the end prosthesis in mind.

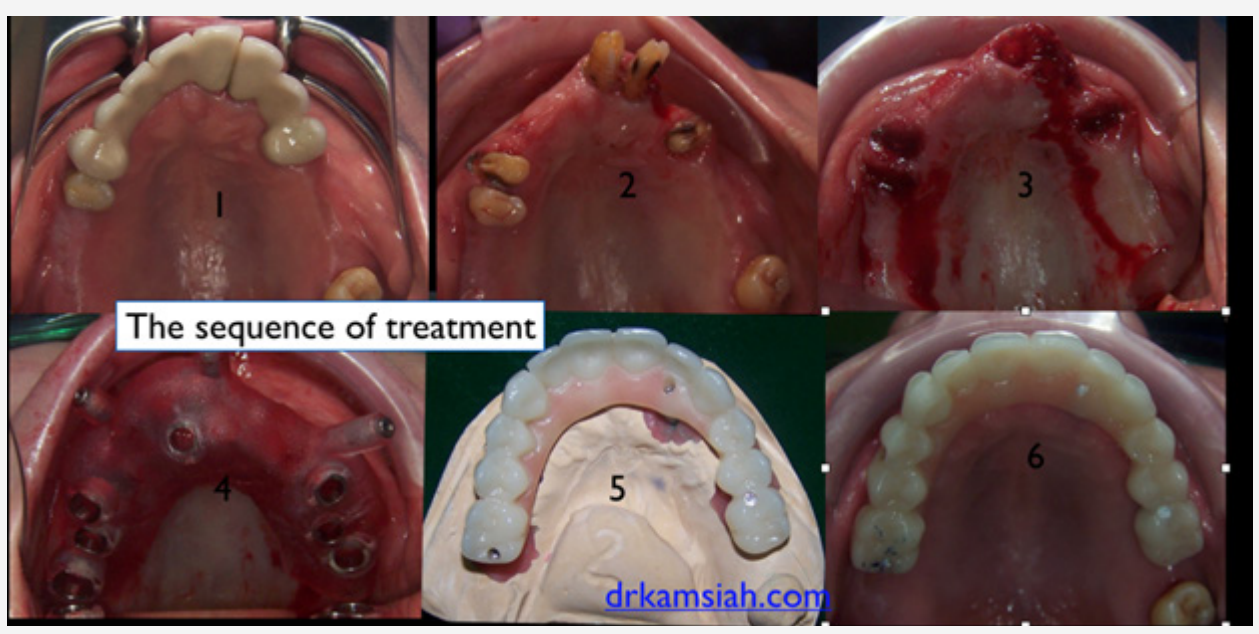

Figure 7: This DSD protocol and AO4 concept allow for an implant supported fixed prosthesis on the same day. 


\section{Combined Pre-treatment Plan with Digital Smile Design}

\section{Case 2}

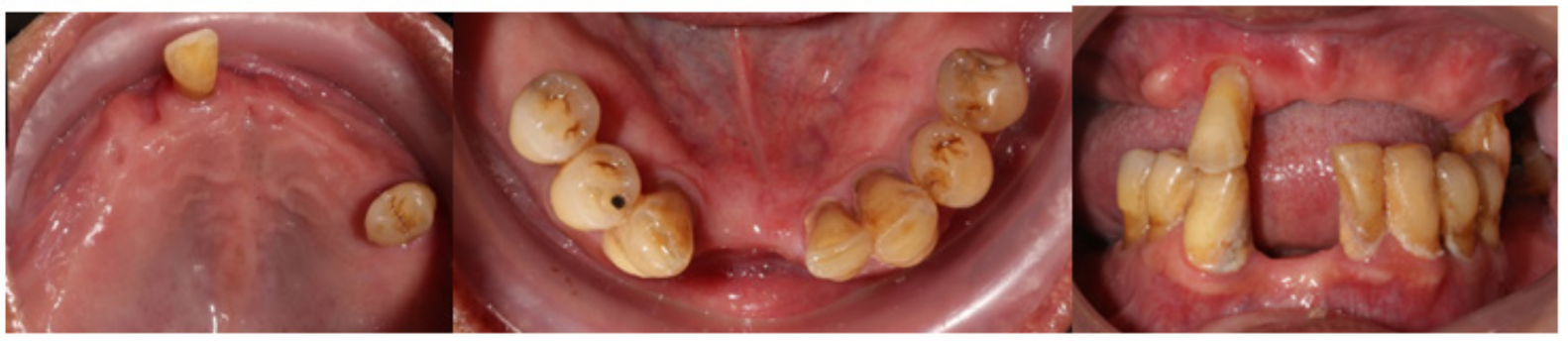

Figure 8: Intraoral Photographs

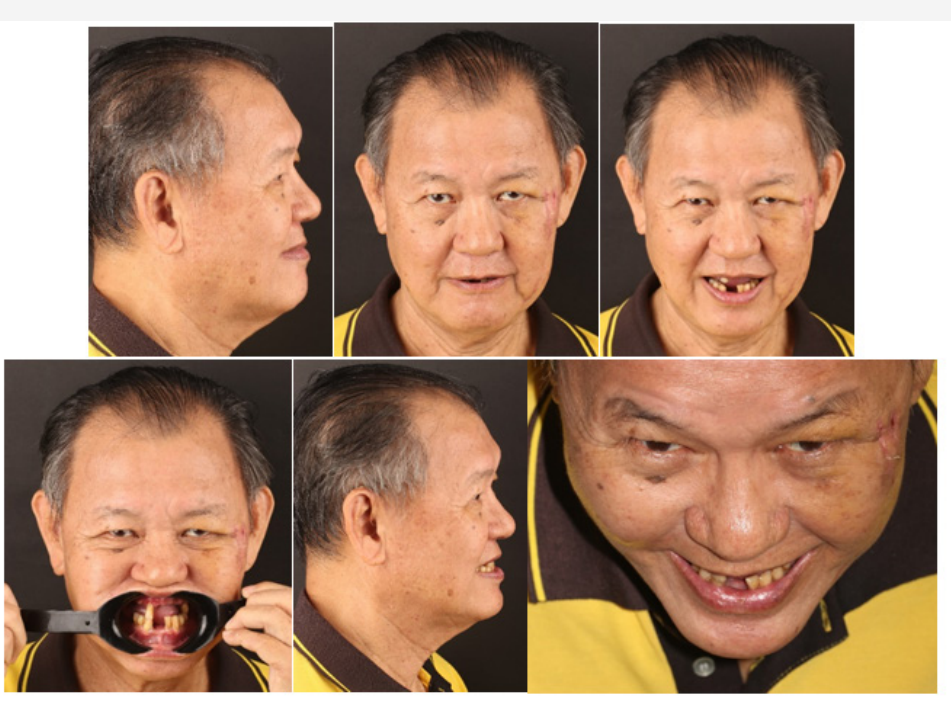

Figure 9: DSD Protocol Photographs without dentures.

A 69-year-old man presented with a maxillary removable partial denture (RPD) and mandibular removable partial denture (RPD). He was unhappy with his removable dentures and expressed strong interest in having fixed permanent teeth (Figures $8 \& 9$ ).

This guided full-arch immediate-function treatment modality with the Digital Smile Design (DSD) digital implant treatment planning solutions is well demonstrated with the following patient. Upon clinical examination and radiographic evaluation, the upper arch showed severe periodontal disease and poor prognosis of remaining teeth. The clinical evaluation included information regarding lip length and support, existing tooth position within the denture and existing teeth, centric and lateral occlusion, restorative space, and speech. Intraoral and extraoral photographs of patient with and without dentures were taken for the purpose of Digital Smile Design (DSD) treatment planning using Keynote. Consent was taken for the usage of patient's personal details and photographs. The DICOM data sets, STL file format, which is a stereolithography CAD software created by 3D Systems, and clinical photographs were then uploaded to the laboratory in Spain, Madrid, using a digital form. In the lab, the patient's STL files were then superimposed with the underlying 3-D bone structures taken from the CBCT scan, forming a complete 3-D patient-specific data set. Using the
DSD protocols, we achieved a complete data set of hard tissue, soft tissue, and biomechanical relationships, by merging the digital impressions, bite, and six clinical photographs, together with the underlying 3-D bony anatomy from the CBCT scan.

Next, by utilizing the digital smile design principle for a harmonic smile, the necessary measures for a complete digital workflow for facially driven dentistry could be accurately determined. A simulated smile of the end result was shown, and the treatment plan was discussed with the patient. A graft less solution was presented to the patient that included full-arch immediate loading in the maxilla, executed via a fully guided immediate-function approach at one appointment, that would include placement of a prefabricated, monolithic PMMA bar-supported maxillary fixed provisional prostheses.

The maxillomandibular relationship was recorded with the help of resin record base and wax occlusion rim. Ultrasonic scaling for the lower arch was done and patient was instructed proper oral hygiene practices including the use of a chlorhexidine mouth rinse before and during the healing process. Any tooth that required removal was extracted. Antibiotic prophylaxis and analgesia were prescribed prior to surgery (Figure 10). 


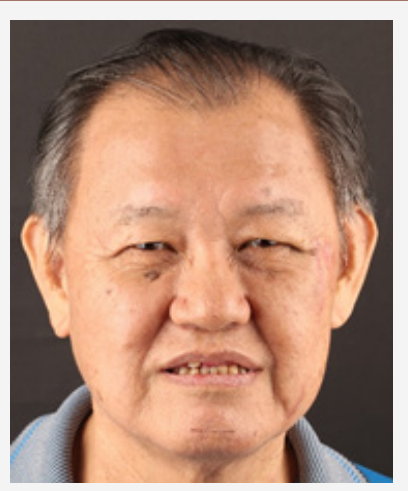

Figure 10: Maxillomandibular relationship using two marks on patient's nose and chin.

On the day of implant placement, the All-on-4 surgical guide was placed in the maxilla arch and secured with three fixation pins while using a bite registration against the opposing natural teeth and RPD to verify its appropriate 3-D position. The two distal implants placed in the posterior region are tilted anterior to the maxillary antrum, at an angle of $30^{\circ}, 3 \mathrm{~mm}$ height. The implant size was $4 \times 15 \mathrm{~mm}$ and drilled till $15 \mathrm{~mm}$. Whereas, the anterior implants was drilled straight at a $5 \mathrm{~mm}$ height till $13 \mathrm{~mm}$. The implant chosen (Dentium) has a design and osseoconductive surface appropriate for ensuring sufficient primary stability for immediate function. The surgical guide and implant mounts were then removed, and the preselected multi-unit abutments were placed and torqued into position according to manufacturer's recommendation. Cone beam computed tomography (СВCT) and periapical radiographs were used to verify full seating of all abutments. A manual torque wrench is used to check the final torque, which should be at least $35 \mathrm{Ncm}$ to allow immediate function (Figure 11).
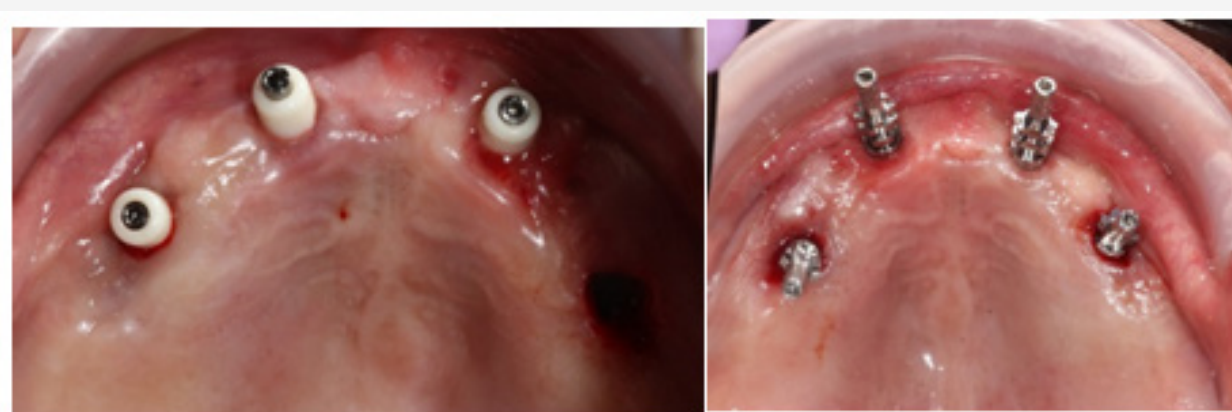

Figure 11: Stress distribution on the Lava restoration along the shoulder margin (a) and Chamfer margin (b).

However, during the 1-month review visit, the implant on 15 failed showing mobilty and patient was having pain, hence a new implant was placed with a bigger diameter of $4.5 \times 12 \mathrm{~mm}$. Three months later, a new impression was taken for the fabrication of the final monolithic PMMA bar-supported maxillary fixed prostheses.

After a month, the upper fixed prosthesis was issued along with the lower removable partial denture. Patient was placed on 20days, 1 month, 3 months and 6 months review (Figures 12 \& 13).

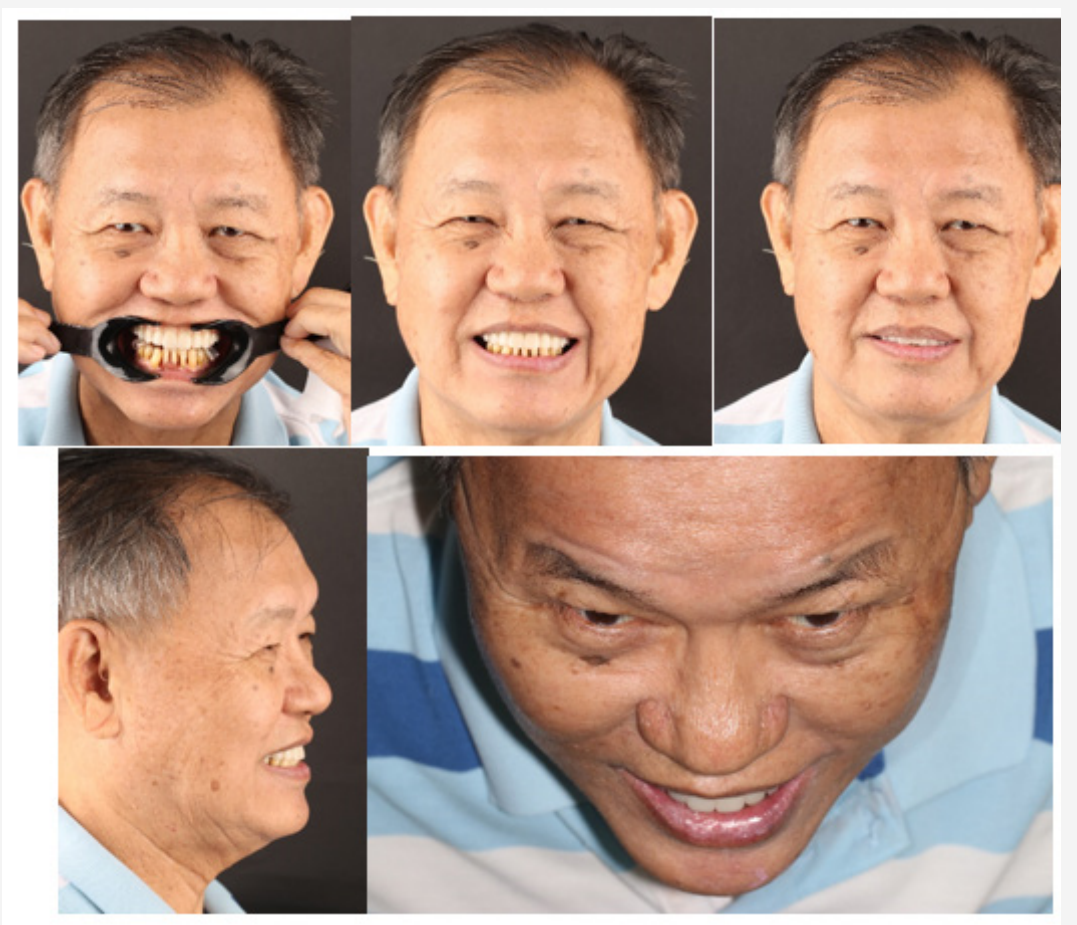

Figure 12: DSD Protocol Clinical Photograph at 20 days review post surgery 


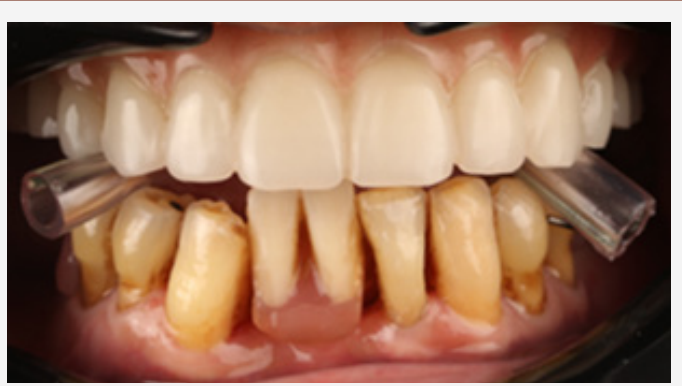

Figure 13: Restored smile.

\section{Conclusion}

This All on four treatment has a long and proven track record to be one of the most predictable in terms of treatment outcome. This procedure has integrated itself as a part of routine treatment plans in our dental clinic because of increasing popularity and patient acceptance. In order to uphold this success, optimum pre-treatment using the DSD protocol is done first together with the diagnosis and even before treatment planning the case. Hence, computerized tomography aided imaging and template-guided surgery plays an important role in pre-placement planning and helps ensure positive outcomes. This article describes the benefits of pre-plan treatment using the DSD protocol, the All on Four concept and computerguided surgery as compared with conventional implant placement procedures, along with a case report.

\section{Acknowledgement}

None.

\section{Conflict of Interest}

Authors declare no conflict of interest.

\section{References}

1. Dawson PE (2007) Functional occlusion from tmd to smile design. 4850.

2. Spear FM (1999) The maxillary central incisor edge A key to esthetic and functional treatment planning. Compend Contin Educ Dent 20(6): 512-516.

3. Kois JC (2002) Diagnostically driven interdisciplinary treatment planning. Seattle Study Club J 6(4): 28-34.

4. Terry DA, Snow SR, Mc Laren EA (2008) Contemporary dental photography: Selection and application. Compend Contin Educ Dent 29(8): 432-440.

5. Coachman C, Van Dooren E, Gurel G, Calamita MA, Calgaro M, et al. (2010) Minimally invasive reconstruction in implant therapy: The prosthetic restoration. Quintessence Dent Technol 33: 61-75.
6. Coachman C, Salama M, Garber D, Calamita M, Salama H, et al. (2009) Prosthetic gingival reconstruction in a fixed partial restoration. Part 1: Introduction to artficial gingiva as an alternative therapy. Int J Periodontics Restorative Dent 29(5): 471-477.

7. Coachman C, Salama M, Garber D, Calamita M, Salama H, et al. (2009) Prosthetic gingival reconstruction in the fixed partial restoration. Part 2: Diagnosis and treatment planning. Int J Periodontics Restorative Dent 29(6): 573-581.

8. Coachman C, Salama M, Garber D, Calamita M, Salama H, et al. (2010) Prosthetic gingival reconstruction in the fixed partial restoration. Part 3. Int J Periodontics Restorative Dent 30(1): 18-29.

9. Goldstein RE (1998) Esthetics in Dentistry Principles, Communication, Treatment Methods. Ontario: Decker.

10. Chiche G, Pinault A (1993) Esthetics of Anterior Fixed Prosthodontics. Quintessence, US, pp. 204-505.

11. Magne P, Belser U Bonded Porcelain Restorations in the Anterior Dentition: A Biomimetic Approach. Chicago.

12. Mittal Y, Jindal G, Garg S (2016) Bone manipulation procedures in dental implants. Indian J Dent 7(2): 86-94.

13. Taruna M, Chittaranjan B, Sudheer N, Tella S, Abusaad M (2014) Prosthodontic Perspective to All- On- $4^{\circledR}$ Concept for Dental Implants. Journal of Clinical and Diagnostic Research. J Clin Diagn Res 8(10): ZE16ZE19.

14. John GP, Joy TE, Mathew J, Kumar VR (2016) Applications of cone beam computed tomography for a prosthodontist. J Indian Prosthodont Soc 16(1): 3-7.

15. Ivanov D, Dol A, Smirnov D (2016) Optimization of dental implant treatment. Russian Open Medical Journal 5(1): e0102.

16. Nagarajan A, Perumalsamy R, Thyagarajan R, Namasivayam A (2014) Diagnostic imaging for dental implant therapy. J Clin Imaging Sci 4(suppl2): 4 .

17. Jivraj S, Chee W (2006) Transitioning patients from teeth to implants. Br Dent J 201(11): 699-708.

18. Jokstad A, Alkumru H (2013) Immediate function on the day of surgery compared with a delayed implant loading process in the mandible: a randomized clinical trial over 5 years. Clin Oral Implants Res 25(12): $1325-1335$.

19. Patel N, Vijayanarayanan R, Pachter D, Coulthard P (2014) Oral healthrelated quality of life: pre- and post-dental implant treatment. Oral Surgery 8(1): 18-22.

20. De Baz C, Hahn J, Lang L, Palomo L (2015) Dental Implant Supported Restorations Improve Quality of Life in Osteoporotic Women. International Journal of Dentistry. Int J Dent pp. 1-6.

21. CK Ho, Dip G, Jovanovic AS (2018) The "All-on-4" Concept for Implant Rehabilitation of an Edentulous Jaw Compendium of Continuing Education in Dentistry.

22. Nik Mastura, Nik Mohammad, Mohamed Yusoff Abbas (2012) Procedia Social and Behavioral Sciences 49: 120-126. 